\title{
Success Begins With Failure: An Alternative Approach in Transfemoral Transcatheter Aortic Valve Replacement Using an Antegrade Wire Crossing Technique
}

\author{
Seshasayee Narasimhan, MBBS, MRCP(UK), FRACP, ${ }^{a}$ Miriam Shanks, MD, FRCPC, a, b \\ Steven Meyer, MD, PhD, FRCSC, ${ }^{\mathrm{a}, \mathrm{b}}$ Benjamin Tyrrell, MD, FRCPC, ${ }^{\mathrm{a}, \mathrm{c}}$ \\ Dylan Taylor, MD, FRCPC, , and Robert C. Welsh, MD, FRCPC $^{\mathrm{a}, \mathrm{b}}$ \\ ${ }^{a}$ Mazankowski Alberta Heart Institute, Edmonton, Alberta, Canada \\ ${ }^{b}$ University of Alberta, Edmonton, Alberta, Canada \\ ${ }^{c}$ CK Hui Heart Centre, Edmonton, Alberta, Canada
}

\begin{abstract}
Transcatheter aortic valve replacement has transitioned from an experimental procedure to an important alternative therapy for patients with symptomatic aortic stenosis and high surgical risk. We present a case outlining an approach to deal with the issue of "failure to cross" the aortic valve in transcatheter aortic valve replacement from the transfemoral retrograde approach.
\end{abstract}

Consistent with the ageing population, degenerative aortic valve stenosis is increasing in prevalence and is one of the most common adult valve conditions seen in the Western world. ${ }^{1}$ It is well documented that conservative management carries a poor prognosis for patients with severe symptomatic aortic stenosis. ${ }^{2}$ Surgical therapy of the calcified aortic valve provides durable and excellent results, ${ }^{3}$ but not all patients are suitable for open heart surgery. Transcatheter aortic valve replacement (TAVR) has become a feasible alternative to open surgical techniques in patients in whom open surgical treatment is contraindicated or who have predictive high risk for perioperative morbidity and mortality.

\section{Case}

A 77-year-old white woman with significant chronic obstructive pulmonary disease with a predicted forced expiratory volume

Received for publication October 22, 2018. Accepted January 13, 2019.

Ethics Statement: The research adhered to ethical guidelines.

Corresponding author: Dr Robert C. Welsh, 2C2 Cardiology, WMC, 8440-112 Street NW, Edmonton, Alberta T6G 2B7, Canada. Tel.: +1-780407-3613; fax: +1-780-407-6452

E-mail: robert.welsh@albertahealthservices.ca

See page 152 for disclosure information.

\section{RÉSUMÉ}

Le remplacement valvulaire aortique par cathéter est passé d'une intervention expérimentale à un traitement alternatif important pour les patients atteints d'une sténose aortique symptomatique et exposés à un risque opératoire élevé. Nous présentons un cas qui décrit une approche pour remédier au problème d'« échec à traverser» la valvule aortique lors du remplacement valvulaire aortique par cathéter selon une approche par voie rétrograde fémorale.

in 1 second of $33 \%$ was reviewed for severe aortic stenosis. Her medical history included hypertension, pulmonary artery hypertension, bilateral pulmonary lobectomies for severe bronchiectasis, osteoporosis, and gastroesophageal reflux disease. Because of the patient's poor forced expiratory volume in 1 second and other comorbidities, she was considered a high-risk candidate for open surgical aortic valve replacement and therefore was referred for consideration of transcatheter aortic valve implantation. The aortic valve area on transthoracic echocardiography was calculated to be $0.54 \mathrm{~cm}^{2}$ with significant valvular calcification. Transesophageal echocardiogram confirmed severely calcified aortic valve leaflets and root with a mean valvular gradient of $52 \mathrm{~mm} \mathrm{Hg}$.

For the procedure, the patient was intubated and ventilated. A 7F sheath was placed in her left femoral vein with insertion of a ventricular pacing wire. A 6F sheath was inserted in her left femoral artery through which a pigtail catheter was placed in the ascending aorta. After right femoral arterial access was obtained, 3 Perclose ProGlide (Abbott Vascular, Abbott Park, IL) arterial closure devices were deployed, and the 22F Edwards sheath was placed in her right femoral artery. Four experienced interventional cardiologists each attempted to cross the aortic valve in retrograde fashion using a variety of coronary catheters over a total duration of 50 minutes without success. A decision was made to proceed with a transseptal approach. A transseptal puncture using an $8 \mathrm{~F}$ Mullins transseptal sheath was undertaken with fluoroscopic and 


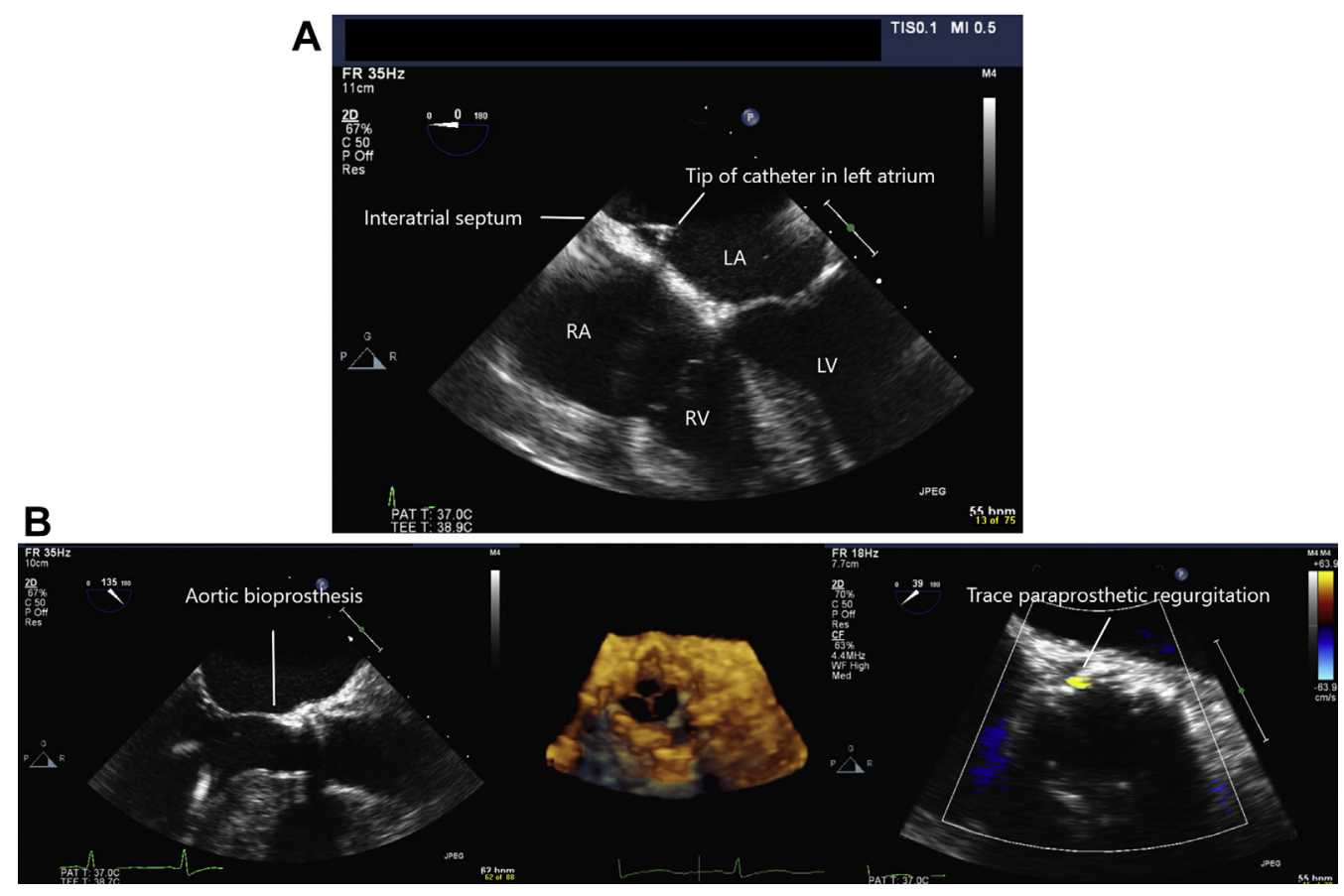

Figure 1. (A) Echocardiography-guided transseptal puncture. The catheter crossing the interatrial septum with its tip in the left atrium is demonstrated on the transesophageal echocardiogram in the midesophageal view at 0 degrees. (B) Intraprocedural echocardiographic assessment of the bioprosthetic valve position and function. Immediately after the valve deployment, transesophageal echocardiogram demonstrated optimal valve position, normally functioning leaflets on 3-dimensional images from the short-axis view, and a trivial paraprosthetic leak on color Doppler. Ao, aorta; LA, left atrium; LV, left ventricle; RA, right atrium; RV, right ventricle.

transesophageal echocardiogram guidance (Fig. 1A). An angle Terumo $(0.035 \times 260)$ wire and a balloon-tipped catheter were advanced through the left atrium, left ventricle, and stenotic aortic valve antegrade into the descending thoracic aorta. Subsequently, a $25-\mathrm{mm}$ snare wire $(120 \mathrm{~cm}$, ev3 Inc., Plymouth, MN) was introduced retrograde into the descending aorta through the femoral sheath to snare the 260$\mathrm{cm}$ exchange length wire (Terumo, Tokyo, Japan). The exchange wire was extracted through the right femoral arterial sheath, and a multipurpose catheter (Johnson and Johnson, New Brunswick, NJ) was passed retrograde back across the aortic valve. The transseptal wire was then removed, and an extra stiff J wire was introduced from the right femoral artery into the left ventricle. After aortic balloon valvuloplasty (Fig. 2), the transseptal catheter was removed, and a 23-mm Edwards Sapien XT valve (Edwards Lifesciences, Irvine, CA) was deployed using the standard technique. Transesophageal echocardiography was used to confirm the position and noted a trivial paravalvular leak with preserved left ventricular systolic function (Fig. 1B). The Edwards sheath was removed, hemostasis was obtained using 3 Perclose ProGlide (Abbott Vascular), and the patient was extubated and transferred to the coronary care unit in stable condition. Although the patient was diagnosed with a minor expressive dysphasia, she had an otherwise uncomplicated periprocedural recovery and was discharged home on day 2 postprocedure.

\section{Discussion}

With the international expansion of TAVR programs and the increased use of this technique to treat elderly patients with symptomatic severe aortic stenosis, there are bound to be cases in which the aortic valve cannot be crossed in the typical retrograde fashion (although rare). The described method allowed the procedure to be completed successfully, avoiding the need to switch to an alternative approach (ie, transapical or subclavian) or to abandon the procedure altogether. This is important because a significant portion of the risk of this procedure is related to obtaining vascular access with the TAVR sheath.

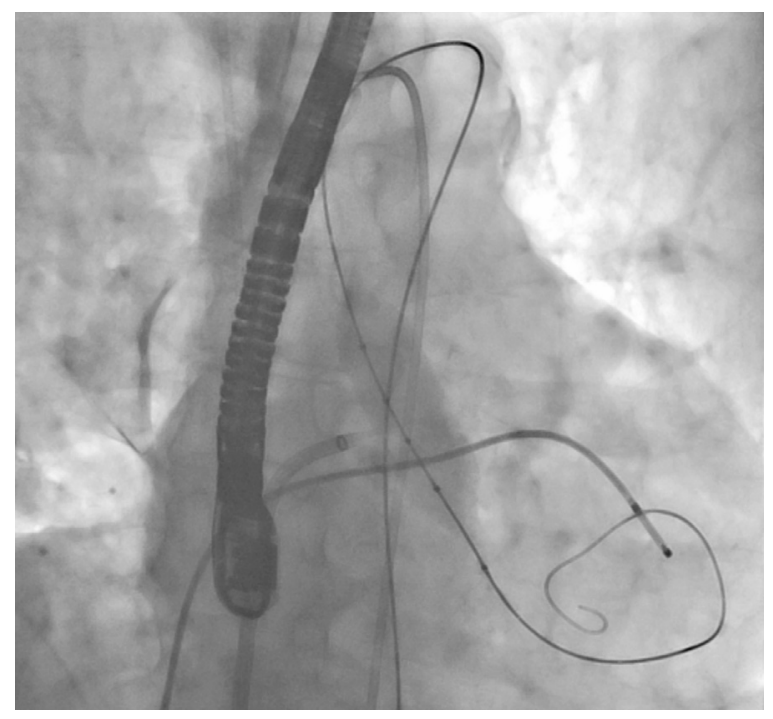

Figure 2. Angiographic image of transseptal puncture and balloon aortic valvuloplasty. 


\section{Conclusion}

Although the described technique was historically used in the era of aortic balloon valvuloplasty and found to be technically and haemodynamically satisfactory compared with the retrograde approach, it has not been reported in association with TAVR. ${ }^{4-6}$ Although this is an uncommon challenge, TAVR heart teams should be aware of this technique to ensure successful procedure completion. In the ideal situation, local TAVR teams have access to onsite expertise to conduct such an approach to allow successful transfemoral TAVR after an initial "failure to cross."

\section{Acknowledgements}

The authors acknowledge the Mazankowski Alberta Heart Institute Transcatheter Aortic Valve Replacement team and Dr John Webb for the dedication to advancing the minimally invasive approach to patients with aortic valve stenosis.

\section{Disclosures}

The authors have no conflicts of interest to disclose.

\section{References}

1. Cowell SJ, Newby DE, Boon NA, et al. Calcific aortic stenosis: same old story? Age Ageing 2004;33:538-44.

2. Varadarajan P, Kapoor N, Bansal RC, et al. Clinical profile and natural history of 453 nonsurgically managed patients with severe aortic stenosis. Ann Thorac Surg 2006;82:2111-5.

3. Lung B, Baron G, Butchart EG, et al. A prospective survey of patients with valvular heart disease in Europe: the Euro Heart Survey on Valvular Heart Disease. Eur Heart J 2003;24:1231-43.

4. Adatia I, Benson LN, Freedom RN. A wire technique to cross the congenitally stenosis aortic valve. Catheter Cardiovasc Diagn 1989;16:250-2.

5. Keane JF, Lock JE. Catheter intervention: balloon valvotomy. In: Lock JE, Keane JF, Fellows KE, eds. Diagnostic and Interventional Catheterization in Congenital Heart Disease. The Hague: Martinus Nijhoff Publishing, 1987:11-122.

6. Block PC, Palacios IF. Comparison of hemodynamic results of anterograde versus retrograde percutaneous balloon aortic valvuloplasty. Am J Cardiol 1987;60:659-62. 